\title{
Research on the Laborers Allocation for the Aircraft Assembly Line Using an Improved Greedy Algorithm
}

\author{
Fangjian Wei, Yu Guo, and Kun Lu
}

\begin{abstract}
As a complex discrete manufacturing system, aircraft assembly line has a lot of different types of laborers. Currently, there are few effective optimization algorithms for laborers allocation problem (LAP). Based on a real aircraft assembly line and its characteristics, the paper proposed an improved greedy algorithm (IGA) which tries to resolve laborer allocation problem. This paper formulates multiple objective functions and constraints according to the actual demand. The utility function is employed to deal with the difficulty of integrating several conflicting and incommensurable objectives into one overall measure. The IGA is an optimization method to achieve balance control of LAP in the discrete manufacturing system based on the core idea of the greedy algorithm. That is to say, optimization can be obtained through moving different operations in a parallel way while the precedence constraints remain unchanged. During the optimization process, 11 attributes are defined for each operation so that the code can be written and the mathematical calculations can be conducted easily. Meanwhile, an example is used to illustrate the optimization process of the algorithm. In this paper, a self-contrast experiment is conducted to verify the validity of the IGA for LAP. The experimental results demonstrate that the IGA can effectively solve the LAP for aircraft assembly line.
\end{abstract}

Index Terms-Aircraft assembly line, optimization, laborer allocation problem (LAP), improved greedy algorithm (IGA).

\section{INTRODUCTION}

Aircraft assembly line is a typically complex discrete manufacturing system, which involves a large number of laborers and operations as well as various types of tools [1]. It belongs to the laborer-intensive production line, especially the final assembly line, which owns a lot of manual work. Its impact on the enterprise depends not only on the quality of the laborers, but also on the laborer utilization. Even though an enterprise owns lots of excellent labors, there won't be high and efficient output without effective laborer utilization. A proper allocation should not only balance the assembly lines, but also improve the efficiency. The LAP can be summed up in one type of assembly line balance (ALB) problem.

In recent decades, the assembly line balancing (ALB) problem has aroused great attention and there have been many researches on it since it was first put forward in the middle of the twentieth century [2]. A lot of concepts, models

Manuscript received July 6, 2015; revised October 12, 2015. This work was supported in part by the Defense Industrial Technology Development Program of China under Grant No.A2520110003.

The authors are with Nanjing University of Aeronautics and Astronautics, 29 Yudao Street, Nanjing 210016, P. R China (e-mail: kenn_wei@ 163.com, guoyu@ nuaa.edu.cn, lukun1991@126.com) and algorithms to solve the ALB problem have been put forward. Generally, the existing ALB literature can be classified into two categories. The first one focuses on classifying problems in the assembly line, such as ALBP-E (Considering the constrained between the number of workstations- $\mathrm{K}$ and production cycle time- $\mathrm{C}$ at the same time and calculating the combination $(\mathrm{K}, \mathrm{C})$ to get the maximum production efficiency); The second category is about the methods of solving the ALB problem. Classical optimization methods include liner programing [3], heuristic procedure [4], dynamic programming [5] and branch and bound algorithm [6]. A large number of advanced intelligent algorithms have also been applied, for example, genetic algorithm [7], ant colony algorithm [8], simulated annealing method [9] and co-evolutionary algorithm [10].

As a subset of ALB, LAP is more completed compared with the traditional ALB problem discussed before. Firstly, it's difficult to define a laborer's behavior because it's very flexible in a laborer-intensive system. Even though there are many performance objectives for a laborer allocation scheme, to evaluate it precisely is a challenge. So far, there has been no closed mathematical formula that can be used to analyze the behavior of laborers in aircraft assembly line. Secondly, the precedence relations of large discrete manufacturing systems are complicated, which have a lot of constraints, but no rules to follow. What's more, for these systems, the allocation scheme is usually allowed to be adjusted according to the real situation. That is to say, for the aircraft assembly line, there is no clear and standard instruction for the operation which is similar to that of the flow shops. Thirdly, as one of the multi-objective optimization problems (MOP), it's also difficult for LAP to deal with the conflicts between incommensurable objectives [11].

One of the earlier studies was done by Hui PCL et al. [12], who proposed a fuzzy operator-allocation system to calculate the number of laborers to be moved in and out of a sewing line. Eryuruk et al. [13] used the heuristic balance algorithm of assembly line to solve multi-model ALB problem in clothing company. Song BL et al. [14] carried out a recursive operator allocation approach for assembly line-balancing optimization problem with the consideration of operator efficiency. There are also some other attempts on the LAP in discrete manufacturing system [15]. But up to now, there has been no proven method for laborers allocation, utilization analysis and objectives evaluation of LAP.

The main purpose of this article is twofold: 1) to present the mathematical formulation of evaluation objectives in aircraft assembly line, 2) to solve the LAPs with IGA efficiently. The research summarizes the constrained relationship based on the characteristics of the aircraft 
assembly line and then proposes four objective functions. The conflicting and incommensurable objectives are solved by the utility function. A new type precedence graph and Gantt chart was devised for the IGA. A self-comparison experiment was designed to validate the effectiveness of this improved algorithm.

The rest of this paper is organized as follows. Section II provides the mathematical formulation of the LAPs. Section III describes the way of IGA to solve the LAPs in details. Section IV validates the effectiveness of this algorithm and analyses the experiment results. Section V is the conclusion and prospect of future study.

\section{FORMULATION OF LABORER AlLOCATION PROBLEM}

\section{A. Notations}

$K$ the number of workstations

$C T$ cycle time of aircraft assembly production line

$d$ the date of assembly line , $1 \leq d \leq C T$

$n_{g} \quad$ the number of elements $\left(n_{1}\right.$ indicates the number of units of a plane; $n_{2}$ refers to the number of technical system groups)

$P_{i} \quad i$ th part of the aircraft, $1 \leq i \leq n_{1}$

$S_{j} \quad j$ th technical system group, $1 \leq j \leq n_{2}$

$O_{i k} k$ th operation in the assembly instruction network diagram of $i$ th part

$L_{j m} \quad m$ th laborer of the $j$ th technical system group

$t_{i k} \quad$ standard allowance time (in hours) of $O_{i k}$, i.e., the time required to complete operation $O_{i k}$ at a defined level of performance

$A T_{j m}^{d}$ all the operating time assigned to $L_{j m}$ in day, $d=1,2, \cdots, C T$

$X_{j m}^{i k}$ the state of operation $O_{i k}$ assigned to laborer $L_{j m}$, if $O_{i k}$ assigned to $L_{j m}$, then is $X_{j m}^{i k}$ equal to 1 ; otherwise, $X_{j m}^{i k}$ is equal to 0

$f_{i} \quad i$ th performance objective function for the aircraft final assembly line, $1 \leq i \leq 4$

$w_{i} \quad i$ th weight of performance objective function $f_{i}$, $1 \leq i \leq 4, \sum_{i=1}^{4} w_{i}=1$

$F$ the overall utility for measure of performance objectives functions

\section{B. Objective Functions}

In ALB problem, there are many characteristic parameters which can reflect the current production situation of the production line, such as the cycle time of production, manufacturing time, waiting time, resource utilization, output and work in process (WIP). The main purpose of ALB is to increase production and reduce the cost. The measure is to reduce the waiting time and improve resource utilization. Aircraft final assembly line is a laborer-intensive production line, so laborers are the main resources. This paper regards people as the priority, emphasizes the allocation scheme of labor and the utilization of all the staff. It also discusses four objective functions.

Objective $1:$ Minimizing the cycle time- $f_{1}$

Cycle time is very important in ABL and many studies have been done. Actually, it's difficult to obtain the accurate CT because of job sharing, operation revisiting, failure and especially the check over aircraft in final assembly line. The cycle time of aircraft final assembly line is defined as:

$$
f_{1}=\min \left\lfloor\frac{\max _{1 \leq i \leq n_{1}} \sum t_{i k}}{8 \times K}\right\rfloor+1
$$

In Eq. (1), the number " 8 " means 8 hours of effective work per day, namely $8: 00$ to $12: 00$ and $14: 00$ to $18: 00$. The number " 1 " is to increase the redundancy of $f_{1}$.

Objective 2: Minimizing total number of laborers- $f_{2}$

As a labor-intensive production line, laborer is one of the main costs for the aircraft final assembly line. Minimizing the number of laborers is one of the methods of reducing the cost. First, an enterprise manager is more concerned about the total number of laborers than the number of laborers in each technical system group. Second, the aircraft final assembly line is a highly discrete production line with each workstation being almost independent. Third, there is a big gap between the workloads of diffident technical systems every day. As mentioned above, the work of more than one technical system will be allocated for the labors, but the staff adjustment is only made within the systems.

$$
f_{2}=\min \sum_{j=1}^{n_{2}} M_{j}
$$

$M_{j}$ refers to the number of laborers in the $j$ th technical system group. The calculation method will be discussed in section III. It should be noticed that perhaps a worker belongs to the present group as well as another one.

Objective 3: Maximize utilization rate of laborers- $f_{3}$

Laborer utilization is a direct evaluation index of allocation optimization scheme. Theoretically, the higher the index is, the better it is. Generally speaking, the time a laborer needs to complete a process is related to many factors, which usually has a certain distribution function. In aircraft assembly line, precise assembly time isn't necessary and an hour's error is permitted. Therefore, we will use the standard working time as the required time to complete a process.

$$
f_{3}=\max \frac{\sum_{j=1}^{n_{2}} \sum_{m=1}^{M_{j}} A T_{j m}^{d}}{8 \times f_{2}}(d=1,2, \cdots, C T)
$$


Objective 4: Minimizing smooth index (SI) of laborer workloads- $f_{4}$

SI is a very important index in ALB, and it is used to evaluate an optimization result. There can be a lot of specific objects of SI, the allocated time for assembling is used to judge whether the workload is fair for each worker. We should notice that the workload here just refers to the working time regardless of the difficulty of the specific content of the work.

$$
f_{4}=\min \left(\sum_{j=1}^{n_{2}} \sum_{m=1}^{M_{j}}\left(A T_{j m}^{d}-8 \times f_{3}\right)^{2}\right)^{\frac{1}{2}}
$$

\section{Overall Utility for Measure of Performance Objective}

4 objective functions are used to choose the best optimization results. However, there is a contradictory relationship among these four functions. For example, if the production $\mathrm{CT}$ is shorter, then it means the number of workers needed and the utilization of workers will increase, but SI will become higher. Since there is no consistency among these four functions, then we must consider their weights in real production.

$$
F=\min \left(w_{1} \times f_{1}+w_{2} \times f_{2}+w_{3} \times f_{3}^{-1}+w_{4} \times f_{4}\right)
$$

$w_{i}$ is determined according to the actual demand. When an aircraft assembly line is running, its stations and the cycle time are determined, so $w_{1}$ should be minimal or equal to 0 . This article mainly consider $f_{2}$ and $f_{3}$, so the $w_{2}$ and $w_{3}$ should be bigger, then is $w_{4}$.

\section{LGA OPTIMIZATION FOR LAPS}

This section introduces the improved greedy algorithm (IGA) to solve LAPs in aircraft assembly line. The architecture of the proposed IGA optimization model for the LAPs is shown in Fig. 1

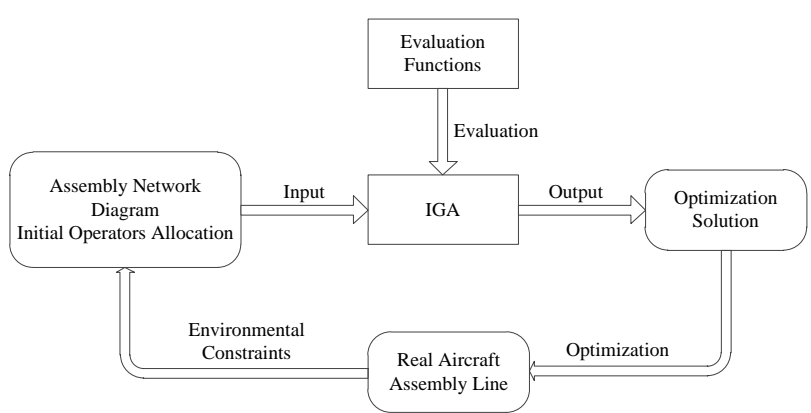

Fig.1. The architecture of IGA optimization for the LAPs.

There are two most important parts in the architecture of IGA optimization model in Fig.1: (1) the improved greedy algorithm to find the optimization solution of LAPs in the aircraft assembly line, (2) the evaluation functions for evaluating each optimization solution to find a better result.
In the front of IGA, we have to ensure the accuracy of the input data, including collecting accurate starting date, precedence constraints, standard operating time and technology group of each operation, drawing accurate assembly network diagram. The most important data is the initial labor allocation scheme because this study focuses on finding a solution to LAPs without considering the performance of this method. As a result, when we do experiment in section IV, the optimization results based on IGA are only compared with the initial allocation scheme rather than other algorithms

\section{A. IGA for Aircraft Assembly Line}

In traditional greedy algorithm, its basic principle is to choose the best strategy in every process. This paper proposes an improved method based on the basic principle of greedy algorithm and the aircraft assembly line. There are two aspects of improvement of greedy algorithm. One is how to choose the optimal strategy for each step of "greedy". In most manufacturing systems, the production line process is relatively simple and "free", which means there is no constrained relationship between it and other resources or environment, and this production line is a single and regular mode, so it's easier to have the greedy selection. However, the actual situation is much more complicated in aircraft assembly line, including the following factors: (1) too many workers for the assembly making it more difficult; (2) too many attributes for each laborer; (3) too many resources need to coordinate in assembly; (4) Each station has multiple parallel parts of the job, but each parallel work location is not absolutely independent and certain constraints are not exist. The other one is how to guarantee the effectiveness of the designed greedy method, because there is not always a link between the local optimal solution and the global optimal solution. We will propose a "translational" approach to solve the multiple constraints mentioned in the first aspect, and make a comparison between the optimization results obtained by IGA with the real laborer allocation in aircraft assembly line. Detailed calculation mechanism of IGA will be described in the following subsections.

\section{1) Representation}

We consider a problem in workstation 1, which has 2 parallel parts, 4 technical system groups, 26 operations and have to be finished within one day. This typical assembly line owns several parallel lines and each parallel line has many serial laborers. Furthermore, nearly every serial line owns different technical system groups. The greedy algorithm does not guarantee the best optimal solution. Therefore, to be consistent with the constraints of the real assembly line and flexibility of the scheme, the final optimization result will be adjusted manually.

\section{2) Data input and initialization}

In this subsection, we do not only input the data, but also generate the precedence graph and Gantt chart of typical problem of the aircraft assembly line. The details of the problem are shown in Fig. 2.

\section{3) Statistics and determine the parallel line}

This subsection mainly statistics on the respective numbers of different technical system groups within the intervals of 
parallel lines, and determines if these parallel operations can compose a new serial line, so that the total operation time of the new serial line is less than 8 hours.

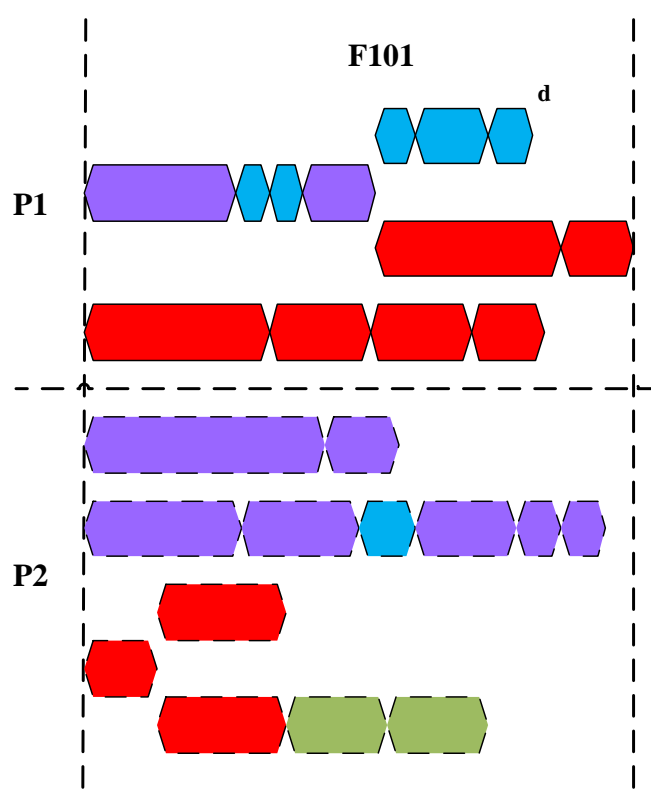

(b). Gantt chart

(a). Precedence graph.

${ }^{\text {a }} \mathrm{P} 1$ represents part 1 of the aircraft and similarly, P2 represents part 2 of the aircraft. The parallel assembly job can be carried out between different parts.

${ }^{\mathrm{b}} \mathrm{F} 101$ refers to the first workstation of the aircraft assembly line in the first day and similarly, F201 means $\mathrm{K}=2, \mathrm{~d}=1$.

${ }^{\mathrm{c}}(2.4 / 4)$ means the standard operation time is 2.4 hours of the operation No.1, and this operation belongs to the 4 th technical system group.

${ }^{\mathrm{d}}$ Gantt chart on the right side corresponds to the PG on the left side, and a polygon box in the Gantt chart represents an operating, and different colors represent different technical system groups, S1=Blue; S2=Red; S3=Green; S4= Purple

Fig. 2. Display of the aircraft assembly line problem.

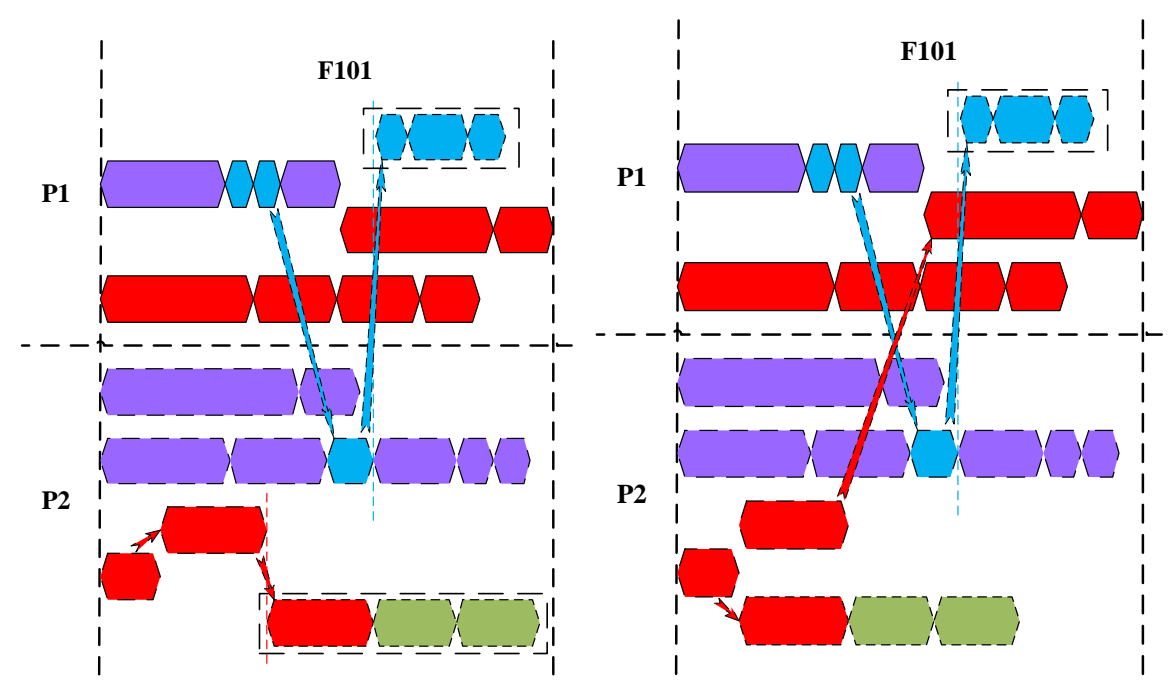

Fig.3. Example of parallel movement.

From Fig. 2 (b), it's easy to find that there are 7 parallel lines in all, and the first technical system group (S1) has 2 parallel lines, S2 has 4 lines, S3 has 1line and S4 has 3 lines. Obviously, some parallel lines belong to the same serial line. This is why when we do parallel movement, must consider the successor and moving current laborer with its successor together. We can also find there indeed has some parallel lines which total operation time is less than 8 hours, that is 6 operations of $\mathrm{S} 1$, which number are 2, 3, 5, 6, 7, 16 and 4 operations of $\mathrm{S} 2$ which number are 8, 9, 22, 3 and 8, 9, 22, 24 and 2 operations of S3. To facilitate the calculation, we use a mathematical approach to define each operation.

Definition 1. Attribute of an operation

We define 11 attributes for each operation, including number (No.), start date (D), workstation (Z), part of aircraft (P), technical system group (S), start time (ST), standard allowance time $(\mathrm{t})$, time coordinate $(\mathrm{X})$, line coordinate $(\mathrm{Y})$, predecessor operation $(\mathrm{F})$ and successor operation $(\mathrm{N})$. The time coordinate $(\mathrm{X})$ refers to the end time of an operation which is equal to the end time of its predecessor operation plus its standard allowance time $(\mathrm{t})$. The line coordinate $(\mathrm{Y})$ refers to longitudinal position coordinate of an operation, and the step between each parallel line is 10 , the step between the serial line and the adjacent parallel line is 5 where the serial line is between two parallel lines, for example, the line coordinate (Y) of No 2, 5, 21 respectively is 5, 0, 40. In addition, the predecessor operation $(\mathrm{F})$ and successor operation $(\mathrm{N})$ may be more than one. Thus, a completed operating property can be expressed as (No., D, Z, P, S, ST, t, 
$\mathrm{X}, \mathrm{Y},(\mathrm{F}),(\mathrm{N}))$, such as the number 15 operations can be expressed as $(15,1,1,2,4,2.5,1.8,4.1,50,(14),(16))$.

\section{4) Parallel movement}

The key to parallel movement is to move with constraints. Fig. 3 displays how to do the parallel movement of LAPs in details.

Step 1: Traverse each technical system group's operation and keep the position of the first operation unchanged.

Step 2: Judge whether there is an overlap between successor operation and predecessor operation in the same group.

Step 3: If any in the step 2, then move the successor operation until there is no overlap with the predecessor. When we move the successor, must keep all the successor operations in the serial line as a whole, such as operations No.5, 6 and 7. If not, keep the position of the operation unchanged.

Step 4: Check if the total standard allowance time of the new serial line is less than 8 hours. If yes, this movement is successful; if not it fails.

In Fig. 3, we can notice that there is more than one way to move the operations, just like (a) and (b). Actually, no matter which method we take, the total number of laborers is the same, both 3 laborers, less than the beginning by one person. As to which way is better, we will discuss in the next subsection.

\section{5) Objective functions evaluation}

As we describe the objective functions in section II, there are different optimization schemes of S4 which are shown in Table I, and there is a principle we must take into consideration. Usually, a serial line is not disconnected so that the same worker can finish the work.

According to discussion, it is clear that the value of functions $f_{1}, f_{2}$ and $f_{3}$ are the same to these four schemes.

Only $f_{4}$ has different values and only the total time will affect

SI. The total time of the three workers is closer, so the SI is smaller. In Table I, compare the total time of scheme 1, 2 and 3 and we can find that the SI of scheme 1 and 2 are the same, smaller than the scheme 3 . Then compare the operations No. allocated to these three laborers of scheme 1 and 2, laborer 1in the scheme 1 do continuous job of $\mathrm{P} 1$, but laborer 1 in the scheme 2 do a new serial job of P1 and P2. Cross-site work is not better than work in the same part, that is scheme 1 is better than scheme 2 .

Scheme 4 is different from the other three schemes. We can clearly see that the SI of scheme 4 is smaller than scheme 1. However, the No. 23 operation allocated to the laborer 3 disconnect from the original serial line, this way contrary to the typically principle, so whether to adopt this optimization method, we should be based on the actual situation, which is manually adjusted in last.

\begin{tabular}{|c|c|c|c|c|c|}
\hline \multicolumn{3}{|c|}{ Scheme 1} & \multicolumn{3}{|c|}{ Scheme 2} \\
\hline laborer & operations No. & $A T_{j m}^{d}(\mathrm{~h})$ & laborers No. & operations No. & $A T_{j m}^{d}(\mathrm{~h})$ \\
\hline L1 & 8,9 & 4 & L1 & $22,8,9$ & 5 \\
\hline L2 & $10,11,12,13$ & 7 & L2 & $10,11,12,13$ & 7 \\
\hline L3 & $22,23,24$ & 5 & L3 & 23,24 & 4 \\
\hline \multicolumn{3}{|c|}{ Scheme 3} & \multicolumn{3}{|c|}{ Scheme 4} \\
\hline laborers No. & operations No. & $A T_{j m}^{d}(\mathrm{~h})$ & laborers No. & operations No. & $A T_{j m}^{d}(\mathrm{~h})$ \\
\hline L1 & $23,8,9$ & 6 & L1 & $22,8,9$ & 5 \\
\hline L2 & $10,11,12,13$ & 7 & L2 & $10,11,12$ & 6 \\
\hline L3 & 22,24 & 3 & L3 & $23,24,13$ & 5 \\
\hline
\end{tabular}

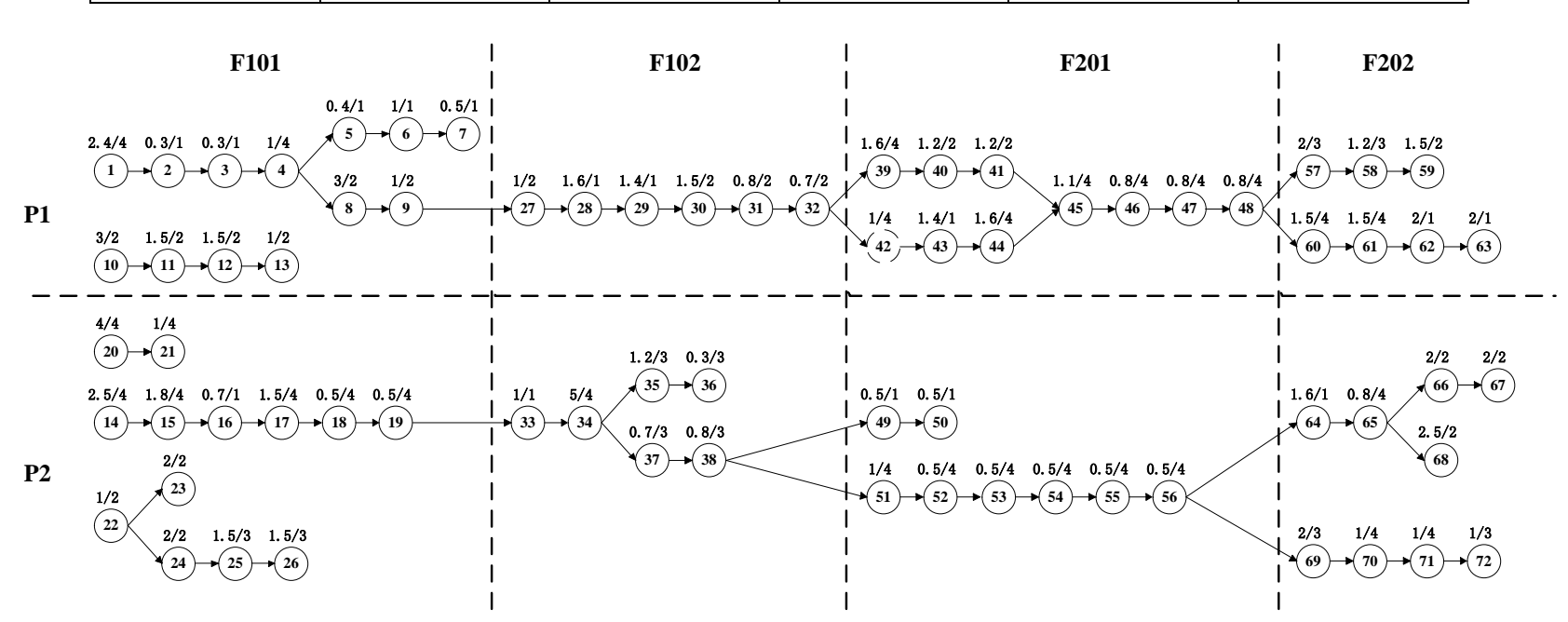

Fig. 4. The experimental precedence graph of an aircraft assembly line. 


\section{B. Manual Adjustment}

The real aircraft assembly line is much more complex without clear constraints so there is no perfect algorithm for LAPs of this production system. However, the ultimate goal of any proposed optimization method is to help enterprises solve practical problems, so we need to optimize the results manually to meet the different needs of different enterprises in the end.

\section{EXPERIMENTS OF VERIFICATION}

In order to verify the validity of the IGA for LAPs, this section will do a self-comparison experiment. That is to say, it is only compared with the original data (OD) to analyze whether the performance of the four objective functions has been improved.

\section{A. Experimental Data}

The data of this experiment is obtained from a real aircraft assembly line, which is shown in Fig.4 and Table II in detail. Totally, there are 72 operations located in 2 workstations and work 2 days. The precedence graph of Fig. 4 has displayed the parallel lines and serial lines clearly, and the length of time interval between two dashed lines is 8 hours. Table II tells us that there are 15 laborers allocated for workstation 1 including 3 laborers in S1, 6 laborers in S2, 2 laborers in S3 and 4 in S4. Meanwhile, 13 laborers are allocated for workstation 2, including 2 in S1, 3 in S2, 2 in S3 and 6 in S4. Table II also shows the operation No. and the total work time allocated for these 28 laborers respectively.

\begin{tabular}{|c|c|c|c|c|c|c|c|c|c|c|c|}
\hline \multirow[b]{2}{*}{$L_{j m}$} & \multirow[b]{2}{*}{$S_{j}$} & \multicolumn{2}{|c|}{ F101 } & \multicolumn{2}{|c|}{ F102 } & \multirow[b]{2}{*}{$L_{j m}$} & \multirow[b]{2}{*}{$S_{j}$} & \multicolumn{2}{|c|}{ F201 } & \multicolumn{2}{|c|}{ F202 } \\
\hline & & $O_{i k}$ & $A T_{j m}^{1}(\mathrm{~h})$ & $O_{i k}$ & $A T_{j m}^{2}(\mathrm{~h})$ & & & $O_{i k}$ & $A T_{j m}^{1}(\mathrm{~h})$ & $O_{i k}$ & $A T_{j m}^{2}(\mathrm{~h})$ \\
\hline L1 & 4 & 1,4 & 3.4 & & 0 & L16 & 4 & 39 & 1.6 & 60,61 & 3 \\
\hline L2 & 1 & 2,3 & 0.6 & 28,29 & 3 & L17 & 2 & 40,41 & 2.4 & 59 & 1.5 \\
\hline L3 & 1 & $5,6,7$ & 1.9 & & 0 & L18 & 4 & 42,44 & 2.6 & & 0 \\
\hline L4 & 2 & 8,9 & 4 & 27 & 1 & L19 & 1 & 43 & 1.4 & 62,63 & 4 \\
\hline L5 & 2 & 10 & 3 & & 0 & L20 & 4 & 45,46 & 1.9 & 65 & 0.8 \\
\hline L6 & 2 & 11,12 & 3 & 30 & 1.5 & L21 & 4 & 47,48 & 1.6 & & 0 \\
\hline L7 & 2 & 13 & 1 & 31,32 & 1.5 & L22 & 1 & 49,50 & 1 & 64 & 1.6 \\
\hline L8 & 4 & 20,21 & 5 & & 0 & L23 & 4 & $51,52,53$ & 2 & & 0 \\
\hline L9 & 4 & 14,15 & 4.3 & & 0 & L24 & 4 & $54,55,56$ & 1.5 & 70,71 & 2 \\
\hline L10 & 1 & 16 & 0.7 & 33 & 1 & L25 & 3 & & 0 & 57,58 & 3.2 \\
\hline L11 & 4 & $17,18,19$ & 2.5 & 34 & 5 & L26 & 2 & & 0 & 66,67 & 4 \\
\hline L12 & 2 & 22,23 & 3 & & 0 & L27 & 2 & & 0 & 68 & 2.5 \\
\hline L13 & 2 & 24 & 2 & & 0 & L28 & 3 & & 0 & 69,72 & 3 \\
\hline L14 & 3 & 25,26 & 3 & 35,36 & 1.5 & & & & & & \\
\hline L15 & 3 & & 0 & 37,38 & 1.5 & & & & & & \\
\hline
\end{tabular}

TABLE III: THE OPTIMIZATION SCHEME FOR EXPERIMENT

\begin{tabular}{|c|c|c|c|c|c|c|c|c|c|c|c|}
\hline \multicolumn{2}{|c|}{} & \multicolumn{2}{|c|}{ F101 } & \multicolumn{2}{c|}{ F102 } & \multicolumn{2}{c|}{ F201 } & \multicolumn{2}{c|}{ F202 } \\
\hline$L_{j m}$ & $S_{j}$ & $O_{i k}$ & $A T_{j m}^{1}(\mathrm{~h})$ & $O_{i k}$ & $A T_{j m}^{2}(\mathrm{~h})$ & $L_{j m}$ & $S_{j}$ & $O_{i k}$ & $A T_{j m}^{1}(\mathrm{~h})$ & $O_{i k}$ & $A T_{j m}^{2}(\mathrm{~h})$ \\
\hline L1 & 4 & 1,4 & 3.4 & 34 & 5 & $\mathrm{~L} 1$ & 4 & $\begin{array}{c}39,51,52,53, \\
54,55,56\end{array}$ & 5.1 & 60,61 & 3 \\
\hline L2 & 1 & $2,3,16,5,6,7$ & 3.2 & $33,28,29$ & 4 & $\mathrm{~L} 2$ & 2 & 40,41 & 2.4 & 59,68 & 4 \\
\hline L3 & 2 & 8,9 & 4 & $27,30,31,32$ & 4 & $\mathrm{~L} 3$ & 4 & $\begin{array}{c}42,44,45, \\
46,47,48\end{array}$ & 6.1 & $65,70,71$ & 2.8 \\
\hline L4 & 2 & $10,11,12,13$ & 7 & & 0 & $\mathrm{~L} 4$ & 1 & $49,50,43$ & 2.4 & $64,62,63$ & 5.6 \\
\hline L5 & 4 & 20,21 & 5 & & 0 & $\mathrm{~L} 5$ & 3 & & 0 & 57,58 & 3.2 \\
\hline L6 & 4 & $14,15,17,18,19$ & 6.8 & & 0 & $\mathrm{~L} 6$ & 2 & & 0 & 66,67 & 4 \\
\hline L7 & 2 & $22,23,24$ & 5 & & 0 & $\mathrm{~L} 7$ & 3 & & 0 & 69,72 & 3 \\
\hline L8 & 3 & 25,26 & 3 & 35,36 & 1.5 & & & & & & \\
\hline L9 & 3 & & 0 & 37,38 & 1.5 & & & & & \\
\hline
\end{tabular}

\section{B. Results and Discussion}

The result calculated by IGA is shown in Table III, including the total number of laborers and operations allocated to these laborers respectively. For the optimization scheme, workstation 1 needs only 9 workers while workstation 2 needs only 7 . Compared with the original data, 
the number of laborers needed decreases $42.9 \%$, which greatly reduces the number of the total workers needed. The comparison charts of the total number and other 3 values of objective functions are shown in Fig. 5 which indicates the results of four objective functions. The cycle time is the same before and after optimization, because the process has finished at the early stage of the design. In addition, the experimental data size is not large enough. It also means the average utilization of laborers with IGA is much higher than original data, for example, $31.17 \%$ of OD and $51.94 \%$ of IGA, nearly $20.00 \%$ growth rate in F101. The remaining average utilizations are $13.33 \%, 15.38 \%$ and $24.62 \%$ of OD, $22.22 \%, 28.57 \%$ and $45.71 \%$ of IGA respectively. The average values are $21.13 \%$ and $37.11 \%$ and growth rate is $16.00 \%$.
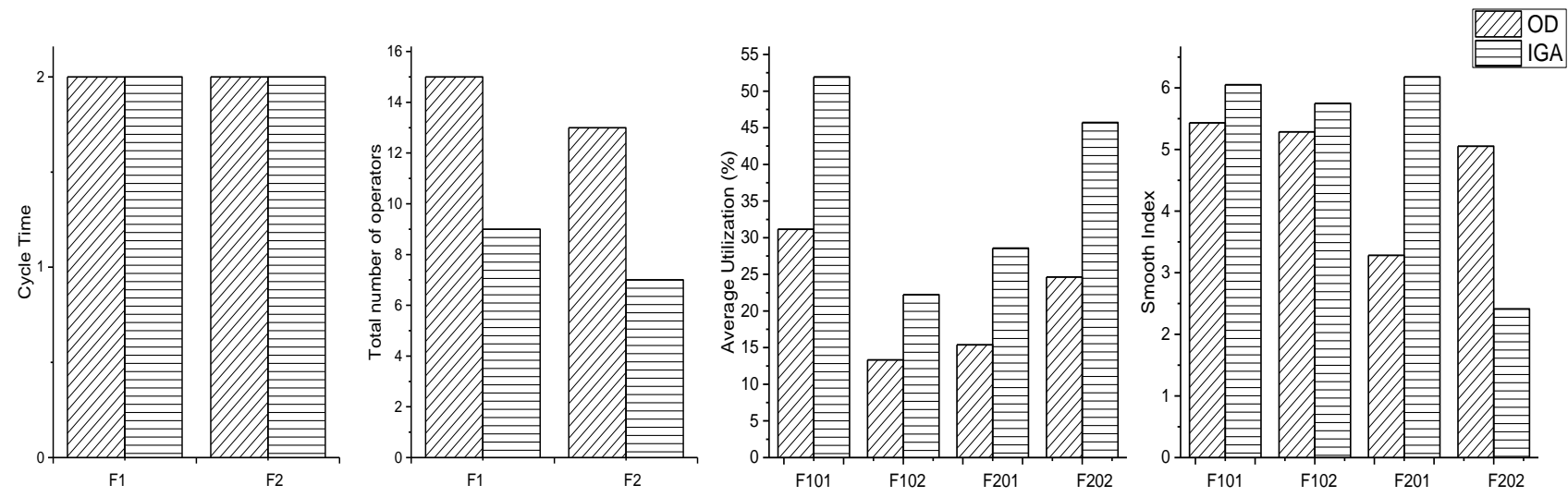

Fig. 5. Experiment results comparison between OD and IGA.

In Fig. 5, it can be seen that there is no rule for smooth index, but the values are higher than before except F202. The values are respectively 5.432, 5.285, 3.281 and 5.053 of OD, $6.052,5.749,6.178$ and 2.412 of IGA. As we all know, managers are more concerned with total number and average utilization of laborers. In subsection II, $w_{i}$ refers to the weight of $f_{i}$. According to the importance of each function, we set $\left(w_{1}, w_{2}, w_{3}, w_{4}\right)$ as $(0.1,0.4,0.4,0.1)$, then obtain the values of $F$ which are shown in Table IV. Obviously, the final results indicate the optimization with improved the greed algorithm (IGA) is effective.

TABLE IV: THE VALUES OF $F$ FOR THE EXPERIMENT

\begin{tabular}{|c|c|c|c|c|}
\hline & F101 & F102 & F201 & F202 \\
\hline OD & 8.026 & 9.729 & 8.329 & 7.530 \\
\hline IGA & 5.175 & 6.175 & 5.018 & 4.116 \\
\hline
\end{tabular}

\section{CONCLUSiOnS}

In this paper, we propose the improved greedy algorithm (IGA) for laborers allocation problems (LAP) in aircraft assembly line. It presents four objective functions and a utility function to deal with conflicting and incommensurable objectives. The detailed steps of IGA are described to display how the structure and logic of IGA run. Meanwhile, a typical example is used for auxiliary instruction. In the end, we design a self-comparison experiment to verify the validity of the IGA for the LAPs. Through the analysis of the results data, we find that the IGA is indeed effective to solve LAPs which can be a new way for enterprise managers to allocated laborers.

Although the IGA is effective for LAPs, further research is necessary if we want to do more for discrete manufacturing system. In this study, only a self-comparison experiment was carried out and much more experiments comparing with other algorithms can be done.

\section{ACKNOWLEDGMENT}

The authors wish to thank Tangjie Zheng (AVIC Hongdu) and Haoshu Cai (NUAA) for their support in preparing this paper.

\section{REFERENCES}

[1] J. Ríos, F. Mas, and J. L. Menéndez, "Aircraft final assembly line balancing and workload smoothing: A methodological analysis," Key Engineering Materials, vol. 502, pp. 19-24, 2012.

[2] P. Nalin, "The assembly line balancing problem: Review articles," Kku Engineering Journal, vol. 8, no. 2, pp. 195-201, 2012.

[3] M. Peeters and Z. Degraeve, "An linear programming based lower bound for the simple assembly line balancing problem," European Journal of Operational Research, vol. 168, no. 3, pp. 716-731, 2012.

[4] S. G. Dimitriadis, "Assembly line balancing and group working: A heuristic procedure for workers' groups operating on the same product and workstation," Computers and Operations Research, vol. 33, pp. 2757-2774, 2006.

[5] J. Bautista and J. Pereira, "A dynamic programming based heuristic for the assembly line balancing problem," European Journal of Operational Research, vol. 194, no. 3, pp. 787-794, 2009.

[6] Y. Bukchin and I. Rabinowitch, "A branch-and-bound based solution approach for the mixed-model assembly line-balancing problem for minimizing stations and task duplication costs," European Journal of Operational Research, vol. 174, no. 1, pp. 492-508, 2006.

[7] Z. X. Guo, "A genetic-algorithm-based optimization model for solving the flexible assembly line balancing problem with work sharing and workstation revisiting," Reviews IEEE Transactions on Systems Man \& Cybernetics Part C Applications, vol. 38, no. 2, pp.218-228, 2008.

[8] P. R. McMullen and P. Tarasewich, "Multi-objective assembly line balancing via a modified ant colony optimization technique," International Journal of Production Research, vol. 44, no. 1, pp. 27-42, 2006.

[9] A. Baykasoglu, "Multi-rule multi-objective simulated annealing algorithm for straight and U type assembly line balancing problems," Journal of Intelligent Manufacturing, vol. 17, no. 2, pp. 217-232, 2006

[10] Y. K. Kim, S. J. Kim, and J. Y. Kim, "Balancing and sequencing mixed-model U-lines with a co-evolutionary algorithm," Production Planning \& Control, vol. 11, no. 8, pp. 754-764, 2000.

[11] A. Rahimi-Vahed and A. H. Mirzaei, "A hybrid multi-objective shuffled frog-leaping algorithm for a mixed-model assembly line sequencing problem," Computers and Industrial Engineering, vol. 53, no. 4 , pp. 642-666, 2007. 
[12] C. L. Hui, "Fuzzy operator allocation for balance control of assembly lines in apparel manufacturing," IEEE Transactions on Engineering Management, vol. 49, no. 2, pp. 173-180, 2002.

[13] S. H. Eryuruk, F. Kalaoglu, and M. Baskak, "Assembly line balancing in a clothing company," Fibres and Textiles in Eastern Europe, vol. 16, no. 1, pp. 93-98, 2008

[14] B. L. Song, "A recursive operator allocation approach for assembly line-balancing optimization problem with the consideration of operator efficiency," Computers and Industrial Engineering, vol. 51, no. 4, pp. 585-608, 2006.

[15] X. H. Yuan, "Application of enhanced discrete differential evolution approach to unit commitment problem," Energy Conversion Management, vol. 50, no. 9, pp. 2449-2456, 2009.

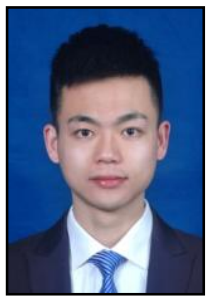

Fangjian Wei is a graduate student of Aeronautics and Astronautics University of Nanjing, China.

$\mathrm{He}$ majores in aeronautics and astronautics manufacturing engineering. His major research is digital design and manufacture.

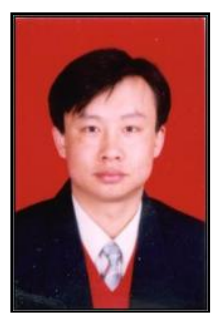

Yu Guo received the Ph.D. degree in mechanical design and its theory from Huazhong University of Science and Technology.

He is a professor of NUAA and the deputy director of the Aerospace Manufacturing Engineering. Now he is working for Nanjing Electrical and Mechanical College of Digital Design Manufacturing Engineering Technology Research Center at the University of Aeronautics and Astronautics and Jiangsu in teaching and research work.

Dr. Guo is a member of the Ministry of Education Changjiang Scholars and Innovative Research Team "advanced aircraft design and manufacture of", and has completed more than 10 defense basic research key projects. He has published more than 50 papers in the domestic and foreign of core journals, writing a monograph, obtained eight eligible software copyrights and 4 patents.

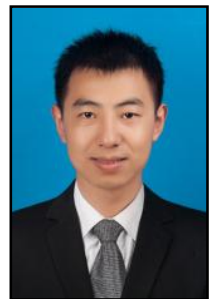

Kun Lu is a graduate student of Aeronautics and Astronautics University of Nanjing, China.

$\mathrm{He}$ majored in aeronautics and astronautics manufacturing engineering. His major research is digital design and manufacture. 